\title{
Voluminoso quiste dentígero de mandíbula tratado en dos etapas quirúrgicas informe de caso
}

\author{
Volumous dentigerous mandibular cyst treated \\ in two surgical steps - case report \\ Volumoso cisto dentígero de mandíbula tratado em \\ duas etapas cirurgicas - relato de caso
}
Sérgio Bartolomeu de Farias Martorelli' ${ }^{1}$ id 0000-0002-0251-1475
Camilo Lellis Carneiro Dias Leite 2 (iD) 0000-0002-1966-6385
Diego Sampaio Garcia Leite ${ }^{2}$ (D) 0000-0003-1429-6351
Marina Rosa Barbosa² (DD 0000-0002-6440-6013
Laís Azevedo Lins de Holanda ${ }^{3}$ (iD) 0000-0001-8967-018X

\section{Resumen}

El quiste dentígero es una lesión benigna que se origina en el epitelio odontogénico asociado a la corona de un diente incluido. Son radiolúcidos y uniloculares, generalmente asintomáticos y diagnosticados en exámenes de rutina o exámenes radiográficos. Los terceros molares inferiores y los caninos superiores son los dientes más afectados, y esta lesión también ocurre en dientes supernumerarios o asociados a odontomas. De crecimiento lento puede alcanzar dimensiones considerables, provocando deformidad facial, impactación y desplazamiento de dientes y/o estructuras adyacentes. El objetivo de este trabajo es informar un caso clínico de un voluminoso quiste dentígero mandibular, que se trató quirúrgicamente en dos etapas: la primera intervención con fines de biopsia y descompresión de la lesión y la segunda con el objetivo de enuclear la cápsula remanente. De esta forma, fue posible realizar un diagnóstico preciso de la lesión, reducir su tamaño para permitir una enucleación total con un daño mínimo a las estructuras anatómicas circundantes y la preservación de la función neurosensorial. Se realizó el seguimiento del paciente durante un período de 5 años en el posoperatorio, y el caso evolucionó hasta la curación total.

Palabras clave: Cirugía bucal; Quiste dentígero; Quistes odontogénicos; Patología bucal.

Cirugía Bucomaxilofacial. Facultad de Odontología de Recife, FOR, FOPCB, Recife, Brasil. smartorelli_maxilofacial@hotmail.com Facultad de Odontología de Recife, FOR, FOPCB, Recife, Brasil.

Odontología Hospitalaria, Instituto de Ciencias Biológicas, Universidad de Pernambuco, Brasil. 


\begin{abstract}
The dentigerous cyst is a benign lesion arising from the odontogenic epithelium associated with the crown of an impacted tooth. They are radiolucent and unilocular, usually asymptomatic and diagnosed in clinical routine examinations or radiographic examination. The lower third molars and upper canines are the most affected teeth and this lesion also occurs associated with odontomas and supernumerary teeth. The cyst has a slow growth but can reach considerable dimensions, causing facial deformity, impaction and displacement of teeth and / or adjacent structures. The aim of this study is to report a clinical case of a large mandibular dentigerous cyst, which was surgically treated in two stages: the first intervention with the purpose of biopsy and decompression of the lesion and a second with the aim of enucleate the cyst capsule. In this way, it was possible to perform an accurate diagnosis of the lesion, reduce its size in order to allow total enucleation with minimal damage to the surrounding anatomical structures, and the preservation of sensitive function. A 5-year follow-up was performed with total lesion healing.
\end{abstract}

Keywords: Oral Surgery; Dentigerous Cyst; Odontogenics Cysts; Oral Pathology.

\section{Introducción}

El quiste dentígero es una lesión intraósea benigna de las más frecuentes dentro de los quistes odontogénicos de desarrollo, asociados principalmente a los terceros molares impactados y caninos superiores, con predisposición en individuos de la segunda a la cuarta década de $\operatorname{vida}^{(1-4)}$. Suelen ser asintomáticos, de creci-

\section{Resumo}

O cisto dentígero é uma lesão benigna oriunda do epitélio odontogênico associado à coroa de um dente incluso. São radiolúcidos e uniloculares, normalmente assintomáticos e diagnosticados em exames de rotina ou exame radiográfico. Os terceiros molares inferiores e os caninos superiores são os dentes mais acometidos, tendo também ocorrência desta lesão em dentes supranumerários ou associados a odontomas. De crescimento lento pode atingir dimensôes consideráveis, causando deformidade facial, impactação e deslocamento de dentes e/ou estruturas adjacentes. O objetivo deste trabalho é relatar um caso clínico de volumoso cisto dentígero mandibular, tratado cirurgicamente em duas etapas: a primeira intervenção com fins de biópsia e descompressão da lesão e uma segunda com intuito de enuclear a capsula rôta. Desta forma pôde-se realizar o diagnostico preciso da lesão, diminuir seu tamanho de forma a permitir a enucleação total com o mínimo de dano as estruturas anatômicas circunvizinhas e a preservação da função neurossensorial. $\mathrm{O}$ paciente foi acompanhado por um período de 05 anos no pós operatório, tendo o caso evoluído para a cura total.

Palavras-chave: Cirurgia Bucal; Cisto Dentígero; Cistos Odontogênicos; Patologia Bucal.

miento lento y se descubren incidentalmente durante la evaluación radiográfica de rutina ${ }^{(5,3)}$. Radiográficamente se caracteriza por ser una lesión radiolúcida unilocular, con bordes escleróticos bien definidos, asociada a la corona del diente impactado ${ }^{(6)}$. Frecuentemente se encuentra la presencia de expansión cortical y reabsorción radicular. Dentro del diagnóstico diferencial se pueden incluir lesiones quísticas, como 
queratoquiste y quiste primordial, y tumores odontogénicos como: ameloblastoma, ameloblastoma unilocular, fibroma ameloblástico y tumor odontogénico adenomatoide ${ }^{(7)}$. Histológicamente, los quistes dentígeros se caracterizan en la mayoría de los casos como una cavidad patológica revestida por un epitelio plano estratificado $^{(5,9)}$. También se puede observar, en algunos casos, la presencia de una cápsula fibrosa de tejido conjuntivo laxo organizado, islotes o hebras de epitelio odontogénico, calcificaciones distróficas y un infiltrado inflamatorio de intensidad variable ${ }^{(9)}$. El tratamiento adoptado en la mayoría de los casos consiste en la enucleación cuidadosa del quiste y la extracción del diente impactado ${ }^{(4,10,11)}$. Los quistes más grandes pueden tratarse mediante marsupialización, facilitando la descompresión y enucleación, mediante un procedimiento quirúrgico electivo conservador $^{(4,5)}$. El pronóstico en la mayoría de los casos es excelente y la recurrencia del quiste es $\operatorname{rara}^{(3,8,12)}$.

\section{Informe de caso}

Paciente afrodescendiente, de sexo masculino, 17 años, estudiante, nacido en Fortaleza, estado de Ceará, en Brasil, que buscó atención en una clínica odontológica privada con quejas de supuración en la boca. Como nos informó la madre, durante aproximadamente un mes el paciente había sentido un mal sabor en la boca. Buscaron un cirujano dentista clínico que, al solicitar una radiografía panorámica de los maxilares, notó una lesión en la mandíbula y lo remitió al cuidado del cirujano bucomaxilofacial. En la anamnesis, no mencionó ninguna queja de dolor: solo mencionó mal sabor en la boca y mal aliento. En el examen físico extrabucal se observaron facies simétricas y ausencia de deformidades faciales. Cadenas ganglionares del complejo cervicofacial sin alteraciones. En el examen intrabucal era visible una supuración en la región del surco gingival lingual y distal 37. Elevación discreta en la región del trígono retromolar izquierdo con punto de fluctuación, de consistencia líquida. Examen de imagen (ortopantomografía) que muestra una extensa lesión osteolítica en la región del ángulo y rama ascendente mandibular izquierda, de aspecto expansivo, asociada al 38 incluido desplazado hacia el hueso basilar (Fig. 1).

Fig. 1: Aspecto radiográfico inicial de la lesión.

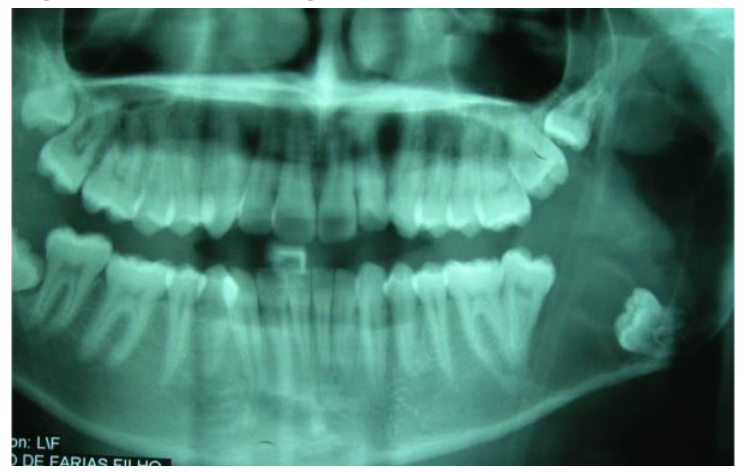

Para una mejor evaluación de la extensión de la lesión, se solicitó una tomografía volumétrica de haz cónico de la región, donde se pudo observar sus límites y relaciones anatómicas circundantes (Fig. 2).

Fig. 2. Tomografía volumétrica de haz cónico permitiendo definir los límites de la lesión.

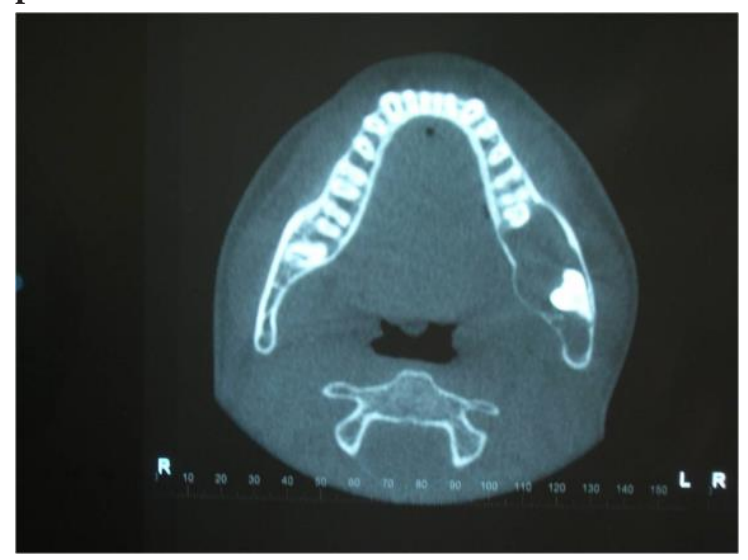

Confrontando la anamnesis, el examen físico y los exámenes complementarios, se estableció la hipótesis diagnóstica de quiste dentígero, ameloblastoma o queratoquiste odontogénico. El tratamiento propuesto y aceptado por los 
responsables luego de leer, concordar y firmar el Formulario de Consentimiento Libre e Informado (FCLI), fue realizar una biopsia de marsupialización bajo anestesia general, con el propósito de diagnosticar y descomprimir la lesión (como terapéutica de retraso). Luego de solicitar los exámenes preoperatorios de rutina, que estaban dentro del estándar normal, se programó la cirugía.

Bajo anestesia general, intubación nasotraqueal, tras antisepsia extra e intrabucal con solución de clorhexidina al 2 y al $0,12 \%$, respectivamente, se realizó la colocación del campo operatorio y el taponamiento del cruce orofaríngeo con "packing». Luego, se realizó una infiltración subperióstica a nivel de la región del trígono retromolar, donde había fluctuación por erosión cortical, con solución de clorhidrato de bupivacaína al 0, 5 \% con hemitartrato de epinefrina 1: 200.000 para promover la hidrodisección perióstica y hemostasia. A continuación, se realizó la aspiración con una jeringa tipo luer de $10 \mathrm{~cm}^{3}$ con aguja 40X12 y se recolectó líquido seropurulento en cantidad abundante (Fig. 3).

Fig.3: Aspiración de contenido seropurulento

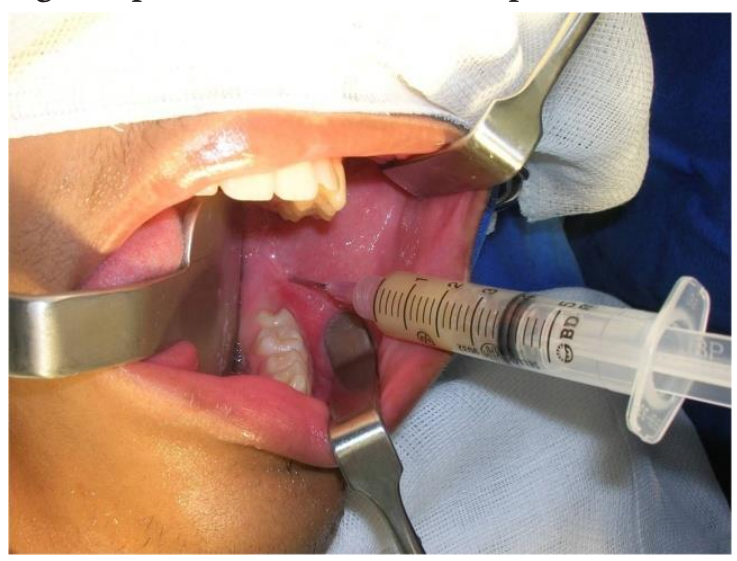

En secuencia, se realizó una incisión circunferencial con un mango para bisturí n.o 3 equipado con una lámina 15 , lo suficientemente profunda para penetrar al interior de la lesión. Además de extraer este tejido, también se ex- trajo un fragmento de la cápsula de la lesión por separado, con el fin de obtener una muestra más específica de la lesión (Fig. 4).

Fig. 4: Extracción de fragmento de la cápsula

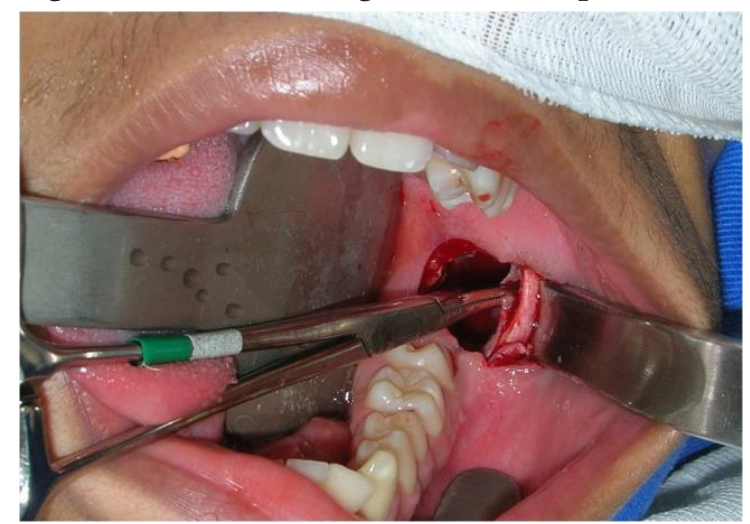

Todo el material extraído se fijó inmediatamente en una solución de formol al $10 \%$. Se realizó una sutura de puntos aislados en toda la zona resecada, abarcando la cápsula de la lesión y la mucosa circundante (Fig. 5). Después de la toilette de la cavidad con suero fisiológico, la cavidad restante se tamponó con una gasa con medicamento impregnada en sulfato de neomicina $5 \mathrm{mg} / \mathrm{g}$ y bacitracina zinc 250 UI pomada.

Fig. 5: Sutura de la cápsula a la mucosa circundante.

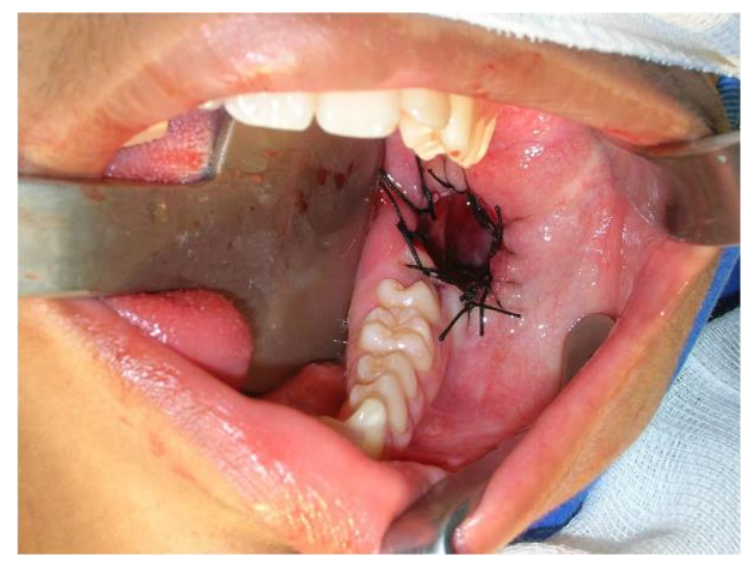

Una semana después, se realizó la extracción de la gasa con medicamento y de la sutura. La cavi- 
dad se mantuvo abierta y se instruyó al paciente y a sus padres sobre la necesidad de realizar una higiene rigurosa con irrigación constante con agua y sustancias antisépticas (enjuagues bucales) durante el período de espera de la regresión de la lesión.

El resultado del examen histopatológico fue compatible con quiste dentígero. Ante el resultado, se decidió esperar a que la regresión de la lesión alcanzara dimensiones compatibles con la mínima posibilidad de daño quirúrgico de las estructuras anatómicas nobles en la región perilesional (paquete neurovascular alveolar inferior y dientes adyacentes).

Un año después de la primera intervención, hubo una clínica y radiográficamente significativa reducción de la lesión (Figs 6 A y 6 B). Entonces se propuso realizar la enucleación de la cápsula remanente del quiste y de los elementos 38, 18, 28 y 48, bajo anestesia general.

Fig. 6 A y 6 B: Aspecto clínico y radiográfico del posoperatorio de 1 año

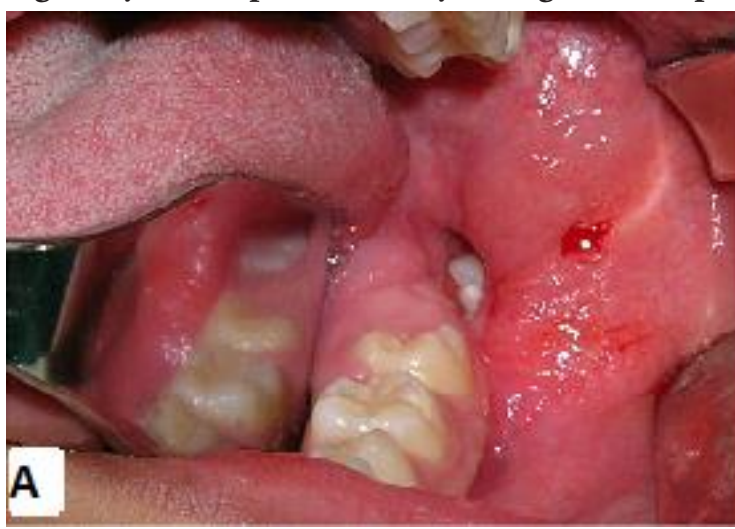

Inicialmente se extrajeron los elementos dentales 18, 28 y 48 mediante técnica quirúrgica tradicional. Luego, se abordó el quiste restante a través de una incisión elipsoide a aproximadamente $0,5 \mathrm{~cm}$ de la abertura del área de descompresión. Tras la enucleación total de la cápsula quística remanente y el elemento 38 (Fig. 7), se realizó el taponamiento de la cavidad con gasa con medicamento impregnada de forma similar a la utilizada en la primera intervención (Fig. 8).

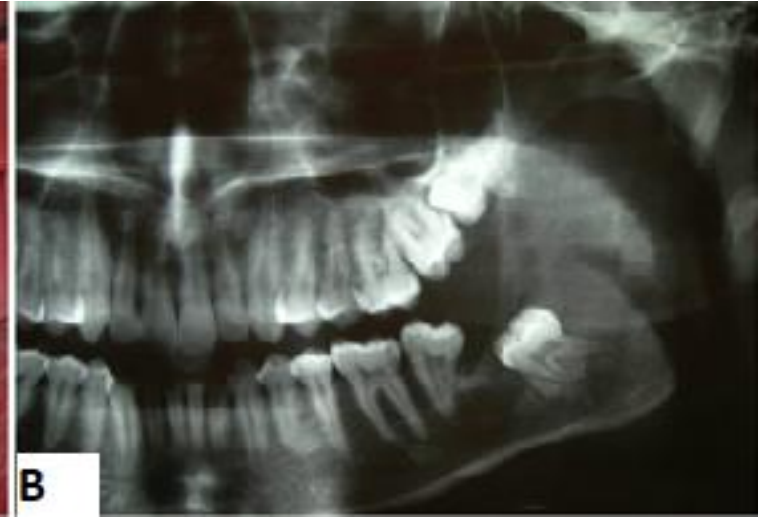

Fig. 7. Extracción de la cápsula quística remanente y del 38.

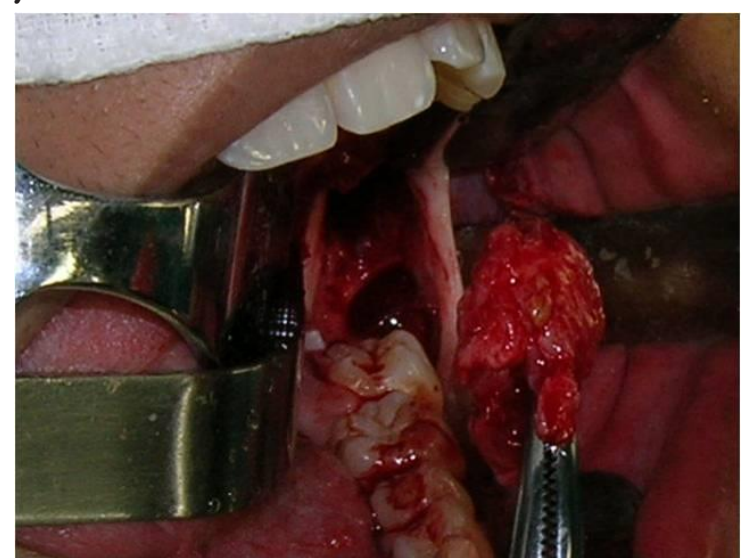


Fig. 8. Taponamiento de la cavidad con gasa con medicamento.

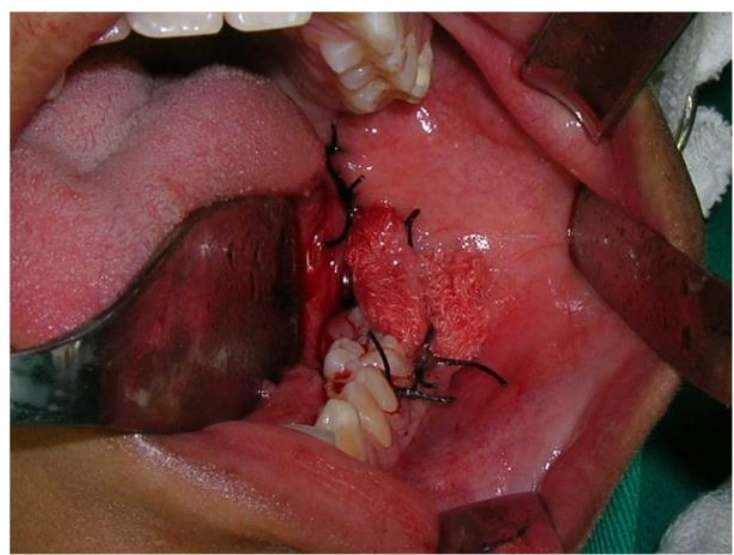

El examen histopatológico de la pieza quirúrgica confirmó definitivamente el diagnóstico inicial de quiste dentígero (Fig. 9).

Fig. 9. Fotomicrografía de la pieza quirúrgica confirmando el diagnóstico definitivo

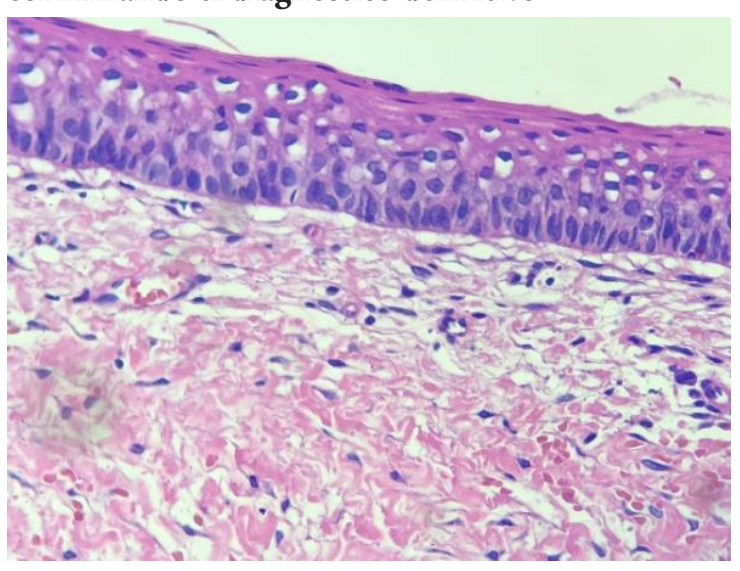

Los puntos se retiraron el 7.0 día de posoperatorio, lo que transcurrió sin ninguna complicación. A diferencia de la primera intervención, donde no hubo exposición de la estructura ósea, en esta ocasión se realizaron los cambios de gasa con medicamento, precedidos de irrigación con suero fisiológico y seguidos de aspiración de la cavidad quirúrgica, semanalmente, hasta observar la completa epitelización de la cavidad quirúrgica, lo que se obtuvo después de la $4 .^{\mathrm{a}}$ semana posoperatoria.

Se realizó el seguimiento del paciente clínica y radiográficamente cada 3 meses durante un período de un año y luego, anualmente, durante un período de cinco años. El aspecto clínico y radiográfico final con 5 años de evolución posoperatoria se puede apreciar en las Fig. $10 \mathrm{~A}$ y Fig. $10 \mathrm{~B}$, donde se observa una completa neoformación ósea.

Tras este período de seguimiento, sin cualquier evidencia clínico-radiográfica de recidiva de la lesión, se dio el alta ambulatoria al paciente.

\section{Discusión}

Por lo visto previamente en la literatura, el quiste dentígero es una lesión intraósea, benigna más frecuente que los quistes odontogéni$\cos ^{(1-4)}$. Normalmente son asintomáticos y de crecimiento lento, se descubren generalmente incidentalmente durante la evaluación clínica y radiográfica de rutina ${ }^{(5,3)}$. Se caracterizan, desde el punto de vista radiográfico, como una lesión

Fig. 10 A y 10 B. Aspecto clínico y radiográfico a los 5 ańos de posoperatorio.

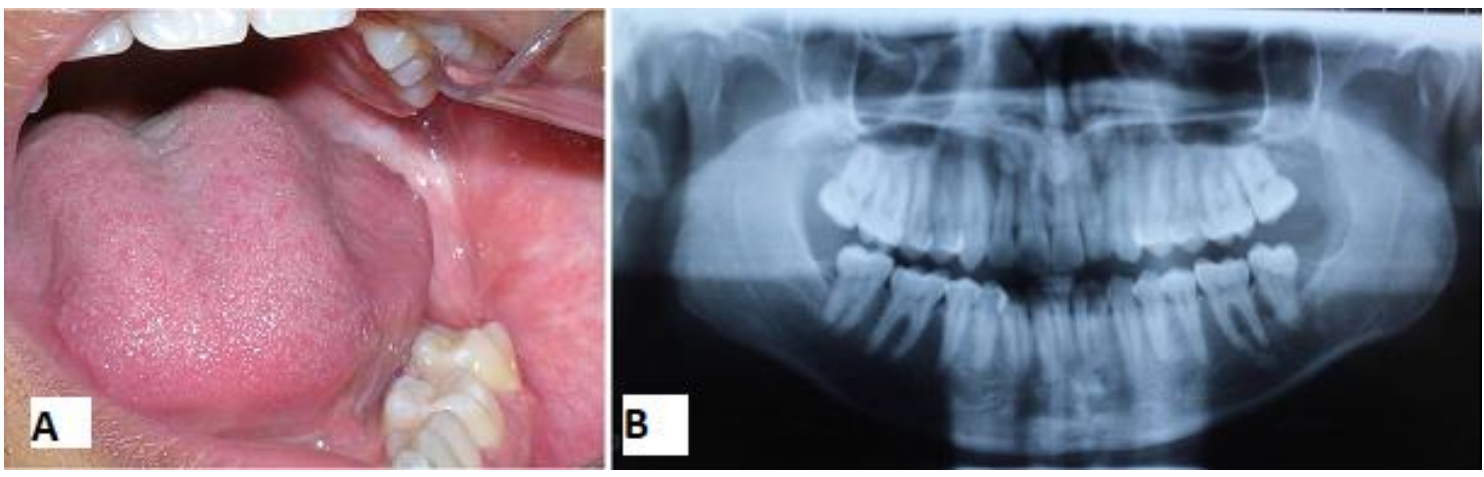


radiolúcida, unilocular, con bordes escleróticos bien definidos, asociada a la corona del diente impactado $^{(6)}$, pero también pueden presentarse de manera sintomática, como se observa en este informe de caso, por la asociación con infección secundaria, donde la supuración fue la queja principal del paciente. La presencia de expansión cortical y reabsorción radicular encontradas con frecuencia, no se encontraba visible clínicamente, lo que por el tamaño de la lesión en este caso no es común.

Histológicamente, los quistes dentígeros se caracterizan en la mayoría de los casos como una cavidad patológica, revestida por un epitelio plano estratificado ${ }^{(5,9)}$, donde en algunos casos específicos también se observa la presencia de una cápsula fibrosa de tejido conectivo laxo organizado, islotes o hebras de epitelio odontogénico, y un infiltrado inflamatorio de intensidad variable $^{(9)}$, lo que es compatible con el espectro histopatológico de este informe de caso.

Coincidimos en que el tratamiento adecuado en la mayoría de los casos consiste en la enucleación cuidadosa del quiste y la extracción del diente impactado ${ }^{(4,10,11)}$, cuando este elemento dental no es viable, lo que permite la extracción in totum de todo el tejido alterado, impidien- do que este tejido sea un proveedor de células tumorales. Los quistes más grandes se pueden tratar mediante descompresión (marsupialización $)^{(4,5)}$, lo que permite un diagnóstico preciso y la descompresión, permitiendo la enucleación de la cápsula remanente posteriormente, sin sacrificar estructuras anatómicas nobles circundantes como el paquete neurovascular alveolar inferior y la vitalidad de dientes contiguos, conducta que se adoptó en este caso en cuestión. El pronóstico en la mayoría de los casos es excelente y la recidiva del quiste es rara $^{3,8,12}$ como se observa en este caso, donde hubo una evolución hasta la curación completa de la lesión.

\section{Conclusión}

En vista de lo que se expuso, se puede concluir que la intervención en dos etapas, en el caso de grandes lesiones quísticas del complejo maxilomandibular, es sumamente valiosa porque permite el diagnóstico preciso del tipo de lesión y minimiza las secuelas de las cirugías, preservando la vitalidad de dientes adyacentes y estructuras anatómicas nobles, como el paquete neurovascular alveolar inferior.

\section{Referencias}

1. Pulido Valladares, Y; Torres Rodríguez, LE; Gounelas Amat, S. Quiste dentígero en la atención pediátrica multidisciplinaria. Rev Ciencias Médicas [Internet]. 2019 Jun [citado 2020 Oct 17] ; 23( 3 ): 473-479.

2. Vasconcelos, MG et al. Localização incomum de cisto dentígero relato de caso. Scientific-Clinical Odontology 2017; 16(4): 200

3. Lacerda, EPM; Martorelli, SBF; Martorlelli, FO. Dentigerous cyst in the maxillary posterior region associated with an impacted deciduous molar. RGO, Rev. Gaúch. Odontol. [Internet]. 2018 June [cited 2020 Oct 15] ; 66( 2 ): 194-198. Available from: http: //www.scielo.br/scielo.php?script=sci arttext\&pid=S1981-86372018000200194\&lng=en

4. Lacerda SHT. Aproveitamento de dentes retidos e associados com cisto dentígero após marsupialização: série de casos. 2015. Revista Intercâmbio 2017; 12: 165

5. Bertolo-Domingues N, Girotto-Bussaneli D, Jeremias Fabiano A, Giro EM, Aguiar-Pansani C. Diagnóstico y tratamiento conservador de quiste dentígero: seguimiento a 3 años. CES odontol. [Internet]. 2018 June [cited 2020 Oct 15] ; 31( 1 ): 57-65. Available from: http: //www.scielo.org.co/scielo. php?script=sci_arttext\&pid=S0120-971X2018000100057\&lng=en. 
6. Morais, HHA et al. Bilateral mandibular dentigerous cysts: a case report. RGO, Rev. Gaúch. Odontol. [Internet]. 2014 Sep [cited 2020 Oct 15] ; 62( 3 ): 299-304. Available from: http: //www.scielo.br/ scielo.php?script=sci_arttext\&pid=S1981-86372014000300299\&lng=en

7. Patil AS, Jathar PN, Panse AM, Bahutule SR, Patil RU, Patil M. Infected Dentigerous Cyst and its Conservative Management: A Report of Two Cases. Int J Clin Pediatr Dent. 2019 Jan-Feb; 12(1): 6872. doi: 10.5005/jp-journals-10005-1578.

8. Spini RG, Bordino L, Cruz D, Fitz Maurice Mde L, Martins A, Michalski J. Quiste dentígero: reporte de un caso [Dentigerous cyst: a case report]. Arch Argent Pediatr. 2016 Oct 1; 114(5): e338-42. doi: 10.5546/aap.2016.e338. Epub 2016 Oct 1.

9. Morales, ND; Díaz Ramos, AG. Quiste dentígero en tercer molar mandibular en posición invertida. Rev Cubana Estomatol [Internet]. 2018 Sep [citado 2020 Oct 17] ; 55( 3 ): 1-8.

10. Lee JY. Dentigerous Cyst Associated With a Supernumerary Tooth. Ear Nose Throat J. 2020 Jan; 99(1): 32-33. doi: 10.1177/0145561318823638. Epub 2019 Jan 28.

11. Sardana D, Goyal A, Gauba K. Manejo de um cisto dentígero inflamado em um paciente com mordida cruzada anterior, usando um obturador modificado. S. Afr. dente. j. [Internet]. Julho de 2016 [citado em 18 de outubro de 2020]; 71 (6): 266-269. Disponível em: http: //www.scielo.org.za/scielo. php?script=sci_arttext

12. Jain N, Gaur G, Chaturvedy V, Verma A. Cisto dentígero associado com pré-molar maxilar impactado: uma ocorrência de local raro e uma coincidência rara. Int J Clin Pediatr Dent. 2018 de janeiro a fevereiro; 11 (1): 50-52. doi: 10.5005 / jp-journals-10005-1483. Epub 1 de fevereiro de 2017.

\section{Nota contribución de los autores:}

1. Concepción y diseño del estudio

2. Adquisición de datos

3. Análisis de datos

4. Discusión de los resultados

5. Redacción del manuscrito

6. Aprobación de la versión final del manuscrito

SBFM ha contribuido en: 1, 2, 3, 4, 5 y 6 .

CLCDL ha contribuido en: 1,2 y 5.

DSGL ha contribuido en: 1, 2 y 5 .

MRB ha contribuido en: 1, 2 y 5 .

LALH ha contribuido en: $1,2,5$ y 6 .

\section{Nota de aceptación:}

Este artículo fue aprobado por la editora de la revista Mag. Dra. Vanesa Pereira-Prado. 